\title{
Excited state dynamics of dGMP measured by steady-state and femtosecond fluorescence spectroscopy
}

\author{
Francois-Alexandre Miannay, Thomas Gustavsson, Akos Banyasz and \\ Dimitra Markovitsi
}

Laboratoire Francis Perrin, CEA/DSM/IRAMIS/SPAM - CNRS URA 2453, CEA Saclay, F-91191 Gif-sur-Yvette, France

AUTHOR EMAIL ADDRESS *: thomas.gustavsson@cea.fr 


\section{Abstract}

The room-temperature fluorescence of 2'-deoxyguanosine 5'-monophosphate (dGMP) in aqueous solution is studied by steady-state and time-resolved fluorescence spectroscopy.

The steady-state fluorescence spectrum of dGMP shows one band centered at $334 \mathrm{~nm}$ but has an extraordinary long red tail, extending beyond $700 \mathrm{~nm}$. Both the fluorescence quantum yield and the relative weight of the $334 \mathrm{~nm}$ peak increase with the excitation wavelength.

The initial fluorescence anisotropy after excitation at $267 \mathrm{~nm}$ is lower than 0.2 for all emission wavelengths indicating an ultrafast $S_{2} \rightarrow S_{1}$ internal conversion. The fluorescence decays depend strongly on the emission wavelength, getting longer with the wavelength. A risetime of 100-150 fs was observed for wavelengths longer than $450 \mathrm{~nm}$, in accordance with a gradual red-shift of the time-resolved spectra.

The results are discussed in terms of a relaxation occurring mainly on the lowest excited ${ }^{1} \pi \pi^{*}$ state surface towards a conical intersection with the ground state in line with recent theoretical predictions. Our results show that the excited state population undergoes a substantial "spreading out" before reaching the CI, explaining the complex dynamics observed.

\section{Keywords}

DNA, nucleotides, dGMP, fluorescence, fluorescence quantum yields, femtosecond spectroscopy, fluorescence upconversion 


\section{Introduction}

There is currently a renewed interest in characterizing optically excited states of DNA and of its constituents ${ }^{1-4}$ and to determine the relevance of these states in the complex mechanisms of DNA photodamage. ${ }^{5}$ However, in order to obtain a detailed understanding of the excited state dynamics of DNA helices, it is crucial to determine the spectroscopy of the monomeric DNA bases. Numerous ultrafast spectroscopic studies in solution have contributed largely to characterize the photophysics of these molecules. Femtosecond transient absorption spectroscopy, ${ }^{6-12}$ fluorescence upconversion, ${ }^{13-20}$ fluorescence Kerr gating ${ }^{21,22}$ and timeresolved infrared spectroscopy ${ }^{23}$ have established that the excited state population decays are globally sub-picosecond, even though there are large differences from on base to another. Fluorescence upconversion measurements performed in our laboratory showed in particular that the excited state relaxation mechanisms of all bases are complex, as manifested by the clearly non-exponential fluorescence decays. ${ }^{14-16,18}$

The detailed understanding of these nonradiative relaxation processes constitutes a major challenge from a theoretical point of view, and an increasing number of quantum chemistry calculations have addressed this problem. The picture emerging is that the ultrafast decay of the excited state is due to highly efficient conical intersections (CI) between the first singlet excited state and the ground state in thymine, ${ }^{24-29}$ cytosine, ${ }^{10,28,30-36}$ and adenine. ${ }^{25,37-48}$

Due to its low solubility in water, guanine is by far the least experimentally studied of the nucleobases. Its nucleoside and nucleotide, on the other hand, have been characterized by ultrafast spectrocopy. ${ }^{7,13,16}$ In ref. 16 it was noted that the steady-state fluorescence spectrum of the guanine chromophore is extraordinarily broad compared to the other nucleosides and nucleotides.

In contrast to experiments, many theoretical studies have been consecrated to the guanine chromophore ${ }^{49-60}$. In line with what was found for the other bases, and the other purine base adenine in particular, the ultrafast decay of the first excited state ${ }^{1} \pi \pi^{*}$ state of the guanine chromophore $\left(\mathrm{L}_{\mathrm{a}}\right.$ or $\mathrm{S}_{1}$ ) was attributed to a practically barrierless path leading from the initially populated state towards a low-lying conical intersection $\left(\mathrm{gs} / \mathrm{S}_{1}\right)_{\mathrm{CI}}$ between the $\mathrm{S}_{1}$ state and the ground state $\mathrm{S}_{0 .}{ }^{52,54,55,57,59}$ All of these calculations considered gas phase conditions. 
The conical intersection $\left(\mathrm{gs} / \mathrm{S}_{1}\right)_{\mathrm{CI}}$ is characterized by a strong geometrical deformation of the purine ring, more precisely a twisting around the $\mathrm{C} 2 \mathrm{~N} 3$ bond provoking an out-of-plane bending of the amino group. The existence of several close-lying conical intersections between the $S_{1}$ state and the ground state $\mathrm{S}_{0}$ involving $\mathrm{N} 1 \mathrm{H}$ deformation ${ }^{57}$ or $\mathrm{C} 2$ pyramidalization ${ }^{59}$ were reported. A second, slower decay path issuing from the second lowest ${ }^{1} \pi \pi^{*}$ state $\left(\mathrm{L}_{\mathrm{b}}\right.$ or $\left.\mathrm{S}_{2}\right)$ and involving relaxation through either the ${ }^{1} n_{N} \pi^{*}$ state ${ }^{52}$ or the ${ }^{1} n_{\mathrm{O}} \pi^{*}$ state ${ }^{55}$ has also been considered. Such a relaxation scheme is in accordance with the bi-exponential excited state decays observed in gas phase experiments. ${ }^{61}$

In a very recent study by Karunakaran et al., ${ }^{62}$ dGMP in aqueous buffer solution was studied by combining femtosecond transient absorption spectroscopy and TD-DFT calculations. The authors concluded that, after UV excitation, ultrafast ${ }^{1}\left(\pi \pi^{*} \mathrm{~L}_{\mathrm{b}}\right) \rightarrow{ }^{1}\left(\pi \pi^{*} \mathrm{~L}_{\mathrm{a}}\right)$ internal conversion occurs (< $100 \mathrm{fs})$, followed by a rapid evolution (0.2 ps) leading from the planar Franck-Condon configuration along a barrierless path on the $\mathrm{L}_{\mathrm{a}}$ state surface to a conical intersection $\left(\mathrm{gs} / \mathrm{S}_{1}\right)_{\mathrm{CI}}$ where internal conversion takes place in 0.9 ps. As in the gas phase, this CI was also characterized by an out-of-plane motion of the amino substituent. The observed bi-exponential excited state dynamics was described as due to the wavepacket motion along a barrierless pathway on the "flat" $\mathrm{L}_{\mathrm{a}}$ state surface only.

In the present paper, we revisit the UV excited states of dGMP in aqueous solution by steadystate and time-resolved fluorescence spectroscopy with higher sensitivity and time resolution than in our previous studies. ${ }^{16}$ In particular, the wavelength range studied is extended all the way into the red spectral region, unraveling a clear wavelength dependence in the fluorescence dynamics. We also note that time-resolved fluorescence measurements provide complementary information compared to transient absorption. It is worth noticing that the excited state dynamics of guaninecontaining organized molecular systems, such as C-G helices ${ }^{63}$ and G-quadruplexes ${ }^{64}$ differ from those of the monomer. Therefore, it is essential to have a more detailed characterization of the guanine chromophore than the one currently available. In this work we only consider the keto-amino form, which was shown to be the only one present in neutral aqueous solution. ${ }^{65}$ 


\section{Experimental Methods}

2'-deoxyguanosine 5'-monophosphate (dGMP) was purchased from Sigma Aldrich and used as delivered. Solutions were prepared in ultrapure water produced by a MILLIPORE (Milli-Q Synthesis) purification system. For control measurements, a phosphate buffer ( $\mathrm{pH}=6.8 ; 0.1 \mathrm{M}$ $\mathrm{NaH}_{2} \mathrm{PO}_{4}, 0.1 \mathrm{M} \mathrm{Na}_{2} \mathrm{HPO}_{4}$ ) containing $0.25 \mathrm{M} \mathrm{NaCl}$ was used to prepare the solutions.

Absorption spectra were recorded with a Perkin-Elmer Lambda 900 spectrophotometer. Fluorescence spectra were recorded with a SPEX Fluorolog-3 spectrofluorometer equipped with a 450 Watt arc Xenon lamp. For both absorption and fluorescence measurements, $1 \mathrm{~cm}$ x $1 \mathrm{~cm}$ quartz cells (QZS) were used.

Fluorescence quantum yields of dGMP in water were measured as a function of the excitation wavelength between 250 and $285 \mathrm{~nm}$. They were indirectly scaled to the previously published value measured for an excitation at $255 \mathrm{~nm}, \Phi_{F}=(1.09 \pm 0.10) \cdot 10^{-4} \cdot{ }^{16}$ Further details are given in the Supporting Information.

Time-resolved fluorescence measurements were made using two different techniques: TimeCorrelated Single Photon Counting (TCSPC) ${ }^{66}$ and fluorescence upconversion (FU). ${ }^{14}$ In both cases, the excitation source was the third harmonic $(267 \mathrm{~nm})$ of a mode-locked Ti-sapphire laser running at $76 \mathrm{MHz}$. The UV pulses were generated in a home-made frequency-tripler using two $0.5 \mathrm{~mm}$ type I BBO crystals. When needed, the laser repetition rate was reduced to $4.75 \mathrm{MHz}$ using a pulse-picker.

For the FU measurements, the average excitation power was set to $40 \mathrm{~mW}$. The fluorescence from the sample is collected by parabolic mirrors and mixed with the residual fundamental in a $0.5 \mathrm{~mm}$ type I BBO crystal in order to generate the sum-frequency. The sum-frequency light is spectrally filtered in a monochromator and detected by a photomultiplier in single-photon counting mode. The spectral resolution of the monochromator at the detection wavelength was set to $8 \mathrm{~nm}$. Parallel $\left(I_{\text {par }}(t)\right)$ and perpendicular $\left(I_{\text {perp }}(t)\right)$ excitation/detection configurations were realized by controlling the polarization of the exciting beam with a zero-order half-wave plate.

Femtosecond fluorescence decays were recorded between 310 and $600 \mathrm{~nm}$ using different filters and crystals. Temporal scans were made in time windows ranging between 2 and 8 ps with corresponding time steps ranging between 13.3 and $100 \mathrm{fs}$ in both parallel and perpendicular configurations. The full width at half maximum (fwhm) of a Gaussian apparatus function is about 
$350 \mathrm{fs}$ at $330 \mathrm{~nm}$ and decreases to about $300 \mathrm{fs}$ at $360 \mathrm{~nm}$ as expected from the GVD mismatch between the fluorescence and the fundamental in the sum-frequency crystal. We judge that the time resolution of our setup is better than $100 \mathrm{fs}$ after deconvolution.

Femtosecond total fluorescence kinetics $F(t)$ were constructed from the parallel and perpendicular signals $\left(I_{p a r}(t)\right.$ and $\left.I_{\text {perp }}(t)\right)$ according to the equation

$$
F(t)=I_{p a r}(t)+2 G I_{p e r p}(t)
$$

where $G$ is the ratio of the sensitivity of the detection system to parallel (vertical) and perpendicularly (vertical) polarized excitation light. In our case $\mathrm{G}$ was measured to 0.95 .

The fluorescence anisotropy decays $r(t)$ were calculated according to the equation

$$
r(t)=\left(I_{\text {par }}(t)-G I_{\text {perp }}(t)\right) / F(t)
$$

Time-resolved fluorescence spectra were recorded by group velocity corrected spectral scans ${ }^{67}$ with $100 \mathrm{fs}$ time steps. For practical reasons, three spectral regions were recorded separately, $300-360,330-450$ and $420-650 \mathrm{~nm}$, using different filters and crystals. The delay stage was corrected for the difference in group velocity during all the scans. The time-integrated spectra were corrected a posteriori with regards to the steady-state fluorescence spectrum and subsequently scaled and joined by using the overlapping regions.

For the FU experiments, solutions $\left(\approx 2.5 \times 10^{-3} \mathrm{~mol} / \mathrm{dm}^{3}\right)$ were kept flowing through a $0.4 \mathrm{~mm}$ quartz cell, which itself was kept in continuous motion perpendicular to the excitation beam. The intensity cannot be measured precisely within the excitation volume but we estimate it to $0.5 \pm 0.1 \mathrm{GW} / \mathrm{cm}^{2}$ for a $40 \mathrm{~mW}$ output at $267 \mathrm{~nm}$ focused to a spot with 30 micron diameter. All time-resolved fluorescence measurements were performed at room temperature $\left(20 \pm 1{ }^{\circ} \mathrm{C}\right)$ under aerated conditions. 


\section{Results}

\section{Steady-state absorption and fluorescence spectra}

The steady-state UV absorption spectrum of dGMP in water along with the fluorescence quantum yields obtained for chosen excitation wavelengths between 250 and $285 \mathrm{~nm}$ are shown in Figure 1.

Fig. 1

The absorption spectrum is characterized by a double-humped structure clearly showing the presence of two electronic transitions at about 270 and $250 \mathrm{~nm}$ in accordance with literature. ${ }^{68-70}$

Table 1

The fluorescence quantum yield decreases when going to shorter excitation wavelengths, indicating more efficient non-radiative deactivation rates at higher excitation energies (see Table 1). Interestingly, this decrease is apparently continuous, in line with the gradually changing overlap between the two electronic transitions involved.

Fig. 2

As shown in Figure 2, the dGMP steady-state fluorescence spectrum has an extraordinary long red wing extending up to $700 \mathrm{~nm}$. This long wavelength tail is much more developed than for the other nucleotides. ${ }^{16}$ The spectra shown in Figure 2 are scaled by the relative fluorescence quantum yield. When decreasing the excitation wavelength, the relative intensity of the red wing remains unchanged while the main peak around $334 \mathrm{~nm}$ decreases linearly with the fluorescence quantum yield. This indicates that the fluorescence spectrum is inhomogeneous, with one part corresponding to the main band around $334 \mathrm{~nm}$ and another part corresponding to the red wing. Rough estimates of these two bands can be obtained by constructing the difference of the spectra resulting from excitation at 255 and $275 \mathrm{~nm}$, normalized either at the peak $(334 \mathrm{~nm})$ or at the red edge $(700 \mathrm{~nm})$. The difference spectra thus obtained are shown in Figure 3. 
Fig. 3

\section{Fluorescence decays}

The TCSPC recordings of dGMP in aqueous solution are completely dominated by an ultrafast component which is limited by the apparatus function (Figure S3 in Supporting Information). No significant contribution at longer times was observed. The residual signal on the nanosecond timescale is proportional to the ultrafast peak and accounts for less than $20 \%$ of the total fluorescence. This information is important for two reasons; first, the residual signal does not influence the early fluorescence dynamics and, second, the time integrated fluorescence spectra discussed below scale directly with the steady-state fluorescence spectrum. Both these points are important when considering the excited state relaxation mechanism. The TCSPC results are not further discussed.

All femtosecond fluorescence decays discussed below were recorded for aqueous solutions of dGMP. We also performed comparative measurements in buffer solution in order to control that the excited state dynamics are not influenced by the ionic strength and/or self-association of dGMP into tetraplex structures. ${ }^{71}$ An example is given in the supporting information. The decays obtained in water and buffer solutions are very similar. This allows us to make direct comparisons between our data obtained in water with lierature data obtained in buffer solution, for example the transient absorption measurements by Karunakaran et al. ${ }^{62}$

In order to verify that no re-excitation of metastable species occurred during the upconversion measurements despite the use of a flow system, comparative experiments with 76 and $4.75 \mathrm{MHz}$ excitation repetition rate were also performed. An example is given in the supporting information. It was found that the fluorescence decays are independent of the excitation repetition rate.

Typical fluorescence decays of dGMP in water as observed in 2 and 8 ps time windows are shown in Figure 4. Also displayed in this figure is the "typical" 330 fs (fwhm) Gaussian apparatus function. It should be kept in mind that the real width of the apparatus function depends slightly on the wavelength as described above.

Fig. 4

The fluorescence decays are strongly wavelength dependent with an apparent fast rise for wavelengths higher than $450 \mathrm{~nm}$. This is in accordance with the important spectral shifts towards 
the red observed in the time-resolved spectra as discussed below. Initially, merged fits of the kinetics for a given fluorescence wavelength were performed. The results are given in Table 2 . The width of the Gaussian apparatus function was free-floating at all wavelengths and shows a complex wavelengths dependence with a minimum at $450 \mathrm{~nm}$. This is in qualitative agreement with the varying interplay between excitation-pulse / fluorescence mismatch in the cell and fluorescence / gate pulse mismatch in the sum-frequency crystal.

Table 2

It was necessary to use a tri-exponential model function at all wavelengths except at $450 \mathrm{~nm}$, where two time-constants, 0.62 and $2.24 \mathrm{ps,} \mathrm{were} \mathrm{obtained.} \mathrm{At} \mathrm{wavelengths} \mathrm{shorter} \mathrm{than} 450 \mathrm{~nm}$, the longest time constant could not be fitted and was artificially fixed to 2.0 ps (arbitrarily chosen as a lower limit for $\tau_{3}$ as judging from the fits at wavelengths longer than $450 \mathrm{~nm}$ ). The smallest time-constant $\left(\tau_{1}\right)$ increases with wavelength, from 0.16 to $0.29 \mathrm{ps,}$, while the intermediate one $\left(\tau_{2}\right)$ decreases very slightly with wavelength, from 0.87 to $0.69 \mathrm{ps}$. At wavelengths longer than $450 \mathrm{~nm}$, a very rapid rise $(0.09-0.17 \mathrm{ps})$ is necessary to obtain an acceptable fit and the slowest time constant $\left(\tau_{3}\right)$ increases with the wavelength, from 2.24 to 3.28 ps.

However, it should be kept in mind that the time constants obtained from the tri-exponential fits are not too different and thus strongly correlated. This implies that the variations in fitted values are not necessarily significant. The strong wavelength dependence is not only due to the different time constants but also to the different pre-exponential factors. Also, as the shorter timeconstants are close to the duration of the apparatus function, this is also a source of correlation.

The main aim with the quantitative fitting procedure is to judge if the time evolution of the fluorescence is caused by an inhomogeneous or a homogeneous spectral relaxation. In the first case, the spectral shift would be caused by the changing intensity balance of two (or several) emission bands, each having a different lifetime. In the latter case, the spectral shift would be due to one emission band moving in time towards longer wavelengths. Consequently, we attempted a global fit using a tri-exponential model function. This fit was satisfactory, giving the timeconstants $(0.16 \pm 0.02),(0.67 \pm 0.07)$ and $(2.0 \pm 0.2)$ ps. Chosen fitted curves are compared to experimental data in Figure 4. As can be seen, the quality is quite satisfactory for all wavelengths. We remark that many more experimental decay curves were included in the global fit than the seven curves shown in Figure 4. 
In order to visualize the spectral distribution of the three terms, Figure 5 shows the decay associated spectra $D A S_{i}(\lambda)$ (with $i=1,2,3$ corresponding to the three exponential terms). These were calculated from the fitted pre-exponential factors according to

$$
\operatorname{DAS}_{i}(\lambda)=\frac{a_{i}(\lambda) * \tau_{i}}{\sum_{j} a_{j}(\lambda) * \tau_{j}} * S S(\lambda)
$$

Where $S S(\lambda)$ is the steady-state fluorescence spectrum (obtained with $267 \mathrm{~nm}$ excitation) which also is shown in Figure 5. The 0.16 ps constant corresponds to a decay up to $450 \mathrm{~nm}$ and a rise from $500 \mathrm{~nm}$ and upwards, while the two longer constants are positive at all wavelengths. The spectral distribution corresponding to the 0.67 ps term resembles the steady-state spectrum while that of the $2.0 \mathrm{ps}$ term is nearly absent in the blue wing and becomes completely dominant in the red wing.

Fig. 5

At this point we note that neither fits at given wavelengths nor a global fit gives a clear picture of the time evolution of the emission band. The spectral relaxation can equally well be due to two different contributions as to one shifting band. This subject will be further discussed in the section on time-resolved fluorescence spectra below.

\section{Fluorescence anisotropy decays}

Fluorescence anisotropy decays of dGMP in water at 330, 420 and $500 \mathrm{~nm}$ are shown in Figure 6. The fluorescence anisotropy decays at other wavelengths show the same behaviour but are not shown here for clarity. A figure illustrating all seven wavelengths is given in the Supporting Information (Figure S5).

Fig. 6

For all wavelengths, the initial anisotropy values $\left(\mathrm{r}_{0}\right.$, at $\left.-0.2 \mathrm{ps}\right)$ are very low in accordance with our earlier reportings. ${ }^{16}$ This indicates an important electronic relaxation at early times, much faster than our time-resolution. Moreover, there is an apparent "slow" decrease at all wavelengths over the 1 ps time window. Finally, going from 310 to 600 nm, a slight decrease can be observed. It should be kept in mind that these traces are convoluted by the apparatus function. 
Consequently, the parallel $\left(I_{\text {par }}(t)\right)$ and perpendicular $\left(I_{\text {perp }}(t)\right)$ recordings were fitted/deconvoluted using a bi-exponential model function for the fluorescence anisotropy as described in the Supporting Information. The two characteristic times for the fluorescence anisotropy decay could not be properly fitted, but diverged towards zero and infinity respectively. They were accordingly fixed to 0.1 and $100 \mathrm{ps}$ in all cases, corresponding to the lower limit of our time-resolution and a "typical" value for the rotational diffusion time of a molecule of the size of dGMP. The fitted initial fluorescence anisotropy values are lower than 0.2 for all wavelengths (se Table S1 in the Supporting Information).

\section{Time-resolved fluorescence spectra}

As described above, the time-resolved spectra shown in Figure 7 were constructed from three separate recordings and scaled relative to the corrected steady-state spectrum obtained with $\lambda_{\text {exc }}=$ $267 \mathrm{~nm}$.

Fig. 7

Inspecting Figure 7, the time-resolved spectra show a significant spectral evolution towards the red. However, care should be taken when viewing this figure since the intensity drops off strongly towards the red, producing the effect of an important "chirp" which is in reality strongly exaggerated. Comparing the occurrence of the temporal "maximum" of the spectrum at 550 and $335 \mathrm{~nm}$, a delay of a about 500 femtosecond can be observed, in line with the 0.16 ps rise deduced from the fits. From Figure 7 it can be seen that the very early time spectra seem to peak around $310 \mathrm{~nm}$, but move towards the steady-state maximum around $334 \mathrm{~nm}$ within a few hundred femtoseconds. Along with this rapid red-shift there is a change in band shape with the development of an important red wing. There is no indication of any inhomogeneous structure in the time-resolved spectra, i.e. two distinct bands, but the $334 \mathrm{~nm}$ peak drops dramatically compared to the red wing. At long times the spectrum adopts a much broader and flatter shape than at early times.

However, displaying the spectra on a wavelength scale is not truly representative when analyzing the spectral shape. Instead we have transformed the spectra to a wavenumber scale, which is directly representative for the energy distribution. 


$$
I(v)=I(\lambda) \cdot \lambda^{2}
$$

Subsequently, in order to quantify the temporal evolution of the spectra, they were fitted by a lognormal function, allowing the calculation of spectral parameters such as the band position, the band width and the spectrally integrated intensity. ${ }^{67}$

The result of this treatment is shown in Figure 8, where corrected time-resolved spectra at chosen delay times together with the corresponding lognormal fits are shown on a wavenumber scale.

Fig. 8

It can be clearly seen how the spectrum not only strongly red-shifts but also broadens rapidly in time. At the longest time, the amplitude has dropped by about $90 \%$ but there is no longer any well defined maximum. The spectrum has attained a practically flat and extremely broad shape covering the whole UV/VIS spectral region.

The corresponding temporal changes in band position and band width are dramatic. Between 0 and $6 \mathrm{ps}$, the mean frequency shifts from about 30000 to less than $20000 \mathrm{~cm}^{-1}$ and the spectral halfwidth increases from about 5000 to about $16000 \mathrm{~cm}^{-1}$. This is shown in Figure S4 in the Supporting Information. These huge changes can be understood in terms of the change of the band profile, from an asymmetric shape with a well-defined maximum at early times to a broader and flatter shape at long times.

\section{Discussion}

The main observations described above for the dGMP photophysics can be summarized as follows. The absorption spectrum is composed of two bands, corresponding to two electronic transitions $\left(S_{0} \rightarrow S_{1}\right.$ and $S_{0} \rightarrow S_{2}$ ) The steady-state fluorescence spectrum is extraordinarily broad and depends on the excitation wavelength while the fluorescence quantum yields increase with increasing excitation wavelength. The femtosecond fluorescence decays depend strongly on the emission wavelength. The time-resolved spectra show an important red-shift correlated with an important broadening. The initial fluorescence anisotropy is lower than 0.20 for all wavelengths. 
Excitation around $267 \mathrm{~nm}$ populates the two first excited ${ }^{1} \pi \pi^{*}$ states $\left(\mathrm{S}_{1}\right.$ and $\left.\mathrm{S}_{2}\right)$ in roughly equal proportions. The question is how this affects the excited state dynamics and in particular the fluorescence. Our measured initial fluorescence anisotropy values are significantly lower than the theoretical limit 0.4 for parallel absorption and emission transition dipoles, indicating that the nature of the emitting state is different from that which absorbs. This electronic relaxation occurs on a timescale which is shorter than our time resolution (100 fs). We may therefore deduce that the $S_{2} \rightarrow S_{1}$ relaxation is much faster than 100 fs. This is very much in line with the findings by Karunakaran et al. ${ }^{62}$

Serrano-Andres et al. ${ }^{55}$ predicted two different pathways for internal conversion to the ground state after UV excitation in gas phase; one fast, direct path from ${ }^{1}\left(\pi \pi^{*} \mathrm{~S}_{1}\right)$ and one slower path from ${ }^{1}\left(\pi \pi^{*} \mathrm{~S}_{2}\right) \rightarrow{ }^{1}\left(\mathrm{n}_{\mathrm{O}} \pi^{*}\right) \rightarrow{ }^{1}\left(\pi \pi^{*} \mathrm{~S}_{1}\right)$ of about $0.8 \mathrm{ps.} \mathrm{From} \mathrm{our} \mathrm{fluorescence} \mathrm{anisotropy} \mathrm{value}$ we have no indications for a slow indirect population of the $S_{1}$ state. The $S_{2} \rightarrow S_{1}$ relaxation seems to be instantaneous. The absence of the indirect path is not surprising since the $n_{\mathrm{O}} \pi^{*}$ state is expected to be strongly destabilized in aqueous solution.

The fact that the fluorescence quantum yield decreases with increasing excitation energy, shows that more efficient non-radiative decay channels open up at higher energies. Increasing the excitation energy implies that the relative $S_{2} / S_{1}$ population ratio increases. Assuming an ultrafast $S_{2} \rightarrow S_{1}$ internal conversion, this will populate higher vibrational $S_{1}$ levels. That is, with increasing excitation energy, an increasingly "hot" $\mathrm{S}_{1}$ population will be the result. In line with the picture of the GMP excited states given by Karunakaran, ${ }^{62}$ it is highly plausible that hotter $\mathrm{S}_{1}$ levels will escape more rapidly from the Franck-Condon region towards the $\left(\mathrm{gs} / \mathrm{S}_{1}\right)_{\mathrm{CI}}$ to undergo internal conversion to the ground state, thus explaining the decreasing fluorescence quantum yield.

In this context it is important to note that not only the fluorescence quantum yield but also the shape of the fluorescence band depends on the excitation wavelength. This is clear from Figure 2, where the fluorescence spectra scaled by the relative fluorescence quantum yield are shown. The fluorescence band is extraordinarily broad for all excitation wavelengths, but while the main peak around $334 \mathrm{~nm}$ decreases with the excitation wavelength the red wing remains more or less constant. This indicates non-negligible fluorescence contributions from a region of the excited state surface with decreased energy gap with regards to the ground state. The inhomogeneous nature of the fluorescence spectrum is emphasized by the difference spectra shown in Figure 3. 
These two bands do not correspond to "absolute" representations of two distinct transitions but rather as an indication of (at least) two contributions to the steady-state fluorescence spectrum.

In line with recent quantum chemistry calculations, ${ }^{62}$ the two bands can tentatively be assigned to the Franck-Condon and local minimum respectively. Interestingly, the population reaching the local minimum is independent of the excitation energy. The decrease of the fluorescence quantum yield observed when increasing the excitation energy affects only the Franck-Condon population and can be seen as an "evaporation" of the hottest part of the $\mathrm{S}_{1}$ population (either directly excited or indirectly populated by $\mathrm{S}_{2} \rightarrow \mathrm{S}_{1}$ conversion) directly towards the (gs/ $\left.\mathrm{S}_{1}\right)_{\mathrm{CI}}$. Thus the population in the shallow local minimum is independent of the excitation energy. It is also possible that new non-radiative relaxation channels open up directly from the $S_{2}$ state, which could explain the decreasing fluorescence quantum yield with excitation energy, since the $\mathrm{S}_{2}$ state is increasingly populated with decreasing excitation wavelength.

Regarding the fluorescence dynamics, the decays depend strongly on the emission wavelength, getting longer when going towards the red. This is very much in contrast with what has been observed for the other nucleotides. All decays are non-exponential, but bi- or tri-exponential fitting, while numerically satisfactory, do not give a clear answer. Individual fits at different wavelength does not produce a common set of time constants, favouring the picture of a continuous shift rather than an inhomogeneous spectral relaxation. However, a global fit with only three time-constants was performed successfully, but this should not be taken as a proof for a sequential two-state relaxation. The shortest decay component of 0.16 ps $\left(\tau_{1}\right)$ dominates in the blue wing but becomes a rise in the red wing. Such a situation can equally well be due to coupled states as a time-dependent spectral relaxation, as remarked by Lakowicz. ${ }^{72}$ This is very much in line with the observed picosecond red-shift of the time-resolved fluorescence spectrum described above.

A dynamical spectral shift would in itself not be surprising; solvation dynamics in water is known to be highly non-exponential, ranging between a few tens of femtoseconds to about a picosecond, ${ }^{73}$ which should cause a spectral shift. Surprisingly, we have not observed any important spectral shifts for the "long-lived" pyrimidine bases, TMP and CMP, under the same conditions. In particular 5-fluorouracil, which has a 1.3 ps average fluorescence lifetime in aqueous solution, did not show any indication of spectral dynamics. ${ }^{18}$ We have observed timedependent spectral shifts for some modified bases, for example, 5-methylcytosine ${ }^{74}$ and 5- 
aminouracil, ${ }^{75}$ but we believe these to be due to multi-state dynamics, i.e. the fluorescence reflects the change in electronic structure possibly related to conformational changes. For these reasons we do not believe that either solvation dynamics or electronic/conformational dynamics are behind the important picosecond spectral relaxation observed for dGMP.

Referring to recent theoretical calculations on dGMP in the gas phase, ${ }^{52,54,55,57,59}$ the internal conversion from the ${ }^{1}\left(\pi \pi^{*} \mathrm{~S}_{1}\right)$ state to the ground state through the $\left(\mathrm{gs} / \mathrm{S}_{1}\right)_{\mathrm{CI}}$ is very fast. Still, when the excited state population moves along the reaction coordinate towards the CI, the closing energy gap would cause a red shift of the fluorescence, but the rapidity of this process would exclude any detectable photon emission. Indeed, the most stable tautomeric forms of guanine, and among them the $9 \mathrm{H}$-amino-keto form, are absent in molecular beam experiments, ${ }^{76}$ which has been attributed to the ultrafast intramolecular non-radiative relaxation processes occurring in the excited state. ${ }^{54}$

Theoretical calculations in solution predicted that the excited state potential energy surface is also practically barrierless and rather flat between the Franck-Condon region and the $\left(\mathrm{gs} / \mathrm{S}_{1}\right)_{\mathrm{Cl}}{ }^{62}$ However, two "pseudominima", a planar one, close to the Franck-Condon region, and a second one, closer to the CI were identified. The flatness of the excited state potential energy surface means that the driving force is small and it is possible that part of the population can be trapped in either. This could explain the initial $334 \mathrm{~nm}$ band and the more slowly appearing red shoulder.

Indeed, the time-resolved spectra displayed on an energy scale show not only a red-shift but rather an important broadening towards a "flatter" structure. This can be described as the diffusion of the excited state population from a relatively localized distribution in the FranckCondon region at early times, to one that is spread out over a much larger region of the excited state at longer times. Such a process implies that the excited state potential energy surface itself is very flat.

We note that Karunakaran et al. ${ }^{62}$ did not observe any wavelength dependence in their transient absorption signals, contrary to our findings. This is not surprising since fluorescence and transient absorption probe different optical transitions. The time-evolution of the fluorescence towards longer wavelengths reflects the decreasing energy gap between the $S_{1}$ and the ground state when the excited population approaches the $\left(\mathrm{gs} / \mathrm{S}_{1}\right)_{\mathrm{CI}}$. Transient absorption reaches a higher excited state of unknown nature for which the energy gap with the $S_{1}$ state may be roughly constant along the reaction coordinate. We not only observe strong wavelength dependence but 
we also measure dramatically longer fluorescence decay times in the red (> $3 \mathrm{ps}$ ) than what was reported by Karunakaran et al. $(0.9 \mathrm{ps}) .{ }^{62}$ Still, we are intrigued by their Figure 9 , where the decay of the $400-900 \mathrm{~nm}$ band integral does not return to zero at $10 \mathrm{ps}$. This indicates a substantial excited state population persisting for much longer times. It is probable that this longlived population becomes non-fluorescent ("dark") beyond a few ps explaining why we do not observe it by fluorescence upconversion.

Finally, it is tempting to draw an analogue to the work by Sundström and co-workers regarding cyanine dyes. $^{77}$ The time-evolution of the excited state population occurring in cyanines along a barrierless reaction path leads to strongly wavelength-dependent fluorescence kinetics. The model of internal conversion through a "sink", reported for the cyanine dyes, is close to the picture proposed for DNA bases, in general, and dGMP, in particular, involving a conical intersection between the first excited singlet state and the ground state. Actually, although the CI represents the point conformation where the ultrafast non-adiabatic internal conversion takes place, the rate for vibrational internal conversion increases rapidly with decreasing energy gap between the $S_{1}$ and $S_{0}$ states allowing a state-switch before the system reaches the CI.

\section{Conclusion}

In this work we have studied the dynamics of the first electronically excited state of dGMP in aqueous solution after excitation at $267 \mathrm{~nm}$. At this wavelength the $S_{1}$ and $S_{2}$ states are populated in roughly equal proportions but, as evidenced from the initial fluorescence anisotropy, an ultrafast electronic relaxation takes place after excitation. This relaxation is too fast to be characterized with our experimental setup. The fluorescence shows a pronounced inhomogeneity, as evidenced both from steady-state and time-resolved spectroscopy.

Steady-state fluorescence spectra and quantum yields depend on the excitation wavelength while the fluorescence decays change with the emission wavelength. This can be explained as the fluorescence emanating from two excited state regions, one close to the Franck-Condon region, and the other along the reaction path leading towards the $\left(\mathrm{gs} / \mathrm{S}_{1}\right)_{\mathrm{Cl}}$, in line with a recent transient absorption / theory work. ${ }^{62}$

In order to confirm this hypothesis it would consequently be very interesting to study the fluorescence decays and time-resolved spectra as a function of excitation wavelength in order to 
see how the excess energy may change the excited state dynamics in terms of initial populations and the branching ratios.

\section{Supporting Information Description}

Description of the Time-Correlated Single Photon Counting (TCSPC) measurements. Description of the fitting/deconvolution procedure of the fluorescence anisotropies. Fluorescence decays at $430 \mathrm{~nm}$ of dGMP in water and buffer solution respectively (Figure S1). Fluorescence decays at $360 \mathrm{~nm}$ of dGMP in water excited with 76 and $4.75 \mathrm{MHz}$ repetition rate respectively (Figure S2). Fluorescence decays at various emission wavelengths of dGMP in water obtained by Time-Correlated Single Photon Counting (Figure S3). Time evolution of the mean frequency and the halfwidth of the dGMP time-resolved fluorescence spectra as obtained by lognorm fitting (Figure S4). Fluorescence anisotropy decays of dGMP in water at 310, 330, 360, 420, 450, 500 and $600 \mathrm{~nm}$ (Figure S5). Fitted initial fluorescence anisotropy values $r_{0}$ at the seven wavelengths (Table S1).

This material is available free of charge via the Internet at http://pubs.acs.org. 


\section{References}

(1) Crespo-Hernández, C. E.; Cohen, B.; Hare, P. M.; Kohler, B. Chem. Rev. 2004,

104, 1977

(2) Crespo-Hernández, C. E.; Cohen, B.; Kohler, B. Nature 2005, 436, 1141.

(3) Markovitsi, D.; Talbot, F.; Gustavsson, T.; Onidas, D.; Lazzarotto, E.; Marguet, S. Nature 2006, 441, E7.

(4) Crespo-Hernández, C. E.; Cohen, B.; Kohler, B. Nature 2006, 441, E8.

(5) Cadet, J.; Sage, E.; Douki, T. Mutat. Res.-Fundam. Mol. Mech. Mutagen. 2005,

$571,3$.

(6) Nikogosyan, D. N.; Angelov, D.; Soep, B.; Lindqvist, L. Chem. Phys. Lett. 1996, $252,322$.

(7) Pecourt, J.-M. L.; Peon, J.; Kohler, B. J. Am. Chem. Soc. 2000, 122, 9348.

(8) Cohen, B.; Hare, P. M.; Kohler, B. J. Am. Chem. Soc. 2003, 125, 13594.

(9) Malone, R. J.; Miller, A. M.; Kohler, B. Photochem. Photobiol. 2003, 77, 158.

(10) Blancafort, L.; Cohen, B.; Hare, P. M.; Kohler, B.; Robb, M. A. J. Phys. Chem. A 2005, 109, 4431. 18641.

(11) Hare, P. M.; Crespo-Hernández , C. E.; Kohler, B. J. Phys. Chem. B 2006, 110,

(12) Hare, P. M.; Crespo-Hernández, C. E.; Kohler, B. Proc. Natl. Acad. Sci. USA 2007, 104, 435 .

(13) Peon, J.; Zewail, A. H. Chem. Phys. Lett. 2001, 348, 255.

(14) Gustavsson, T.; Sharonov, A.; Markovitsi, D. Chem. Phys. Lett. 2002, 351, 195.

(15) Gustavsson, T.; Sharonov, A.; Onidas, D.; Markovitsi, D. Chem. Phys. Lett. 2002, $356,49$.

(16) Onidas, D.; Markovitsi, D.; Marguet, S.; Sharonov, A.; Gustavsson, T. J. Phys. Chem. B 2002, 106, 11367.

(17) Sharonov, A.; Gustavsson, T.; Carré, V.; Renault, E.; Markovitsi, D. Chem. Phys. Lett. 2003, 380, 173.

(18) Gustavsson, T.; Banyasz, A.; Lazzarotto, E.; Markovitsi, D.; Scalmani, G.; Frisch, M. J.; Barone, V.; Improta, R. J. Am. Chem. Soc. 2006, 128, 607.

(19) Pancur, T.; Schwalb, N. K.; Renth, F.; Temps, F. Chem. Phys. 2005, 313, 199.

(20) Schwalb, N. K.; Temps, F. Phys. Chem. Chem. Phys. 2006, 8, 5229

(21) Kwok, W.-M.; Ma, C.; Phillips, D. L. J. Am. Chem. Soc. 2006, 128, 11894.

(22) Kwok, W.-M.; Ma, C.; Phillips, D. L. J. Am. Chem. Soc. 2008, 130, 5131.

(23) McGovern, D. A.; Doorley, G. W.; Whelan, A. M.; Parker, A. W.; Towrie, M.;

Kelly, J. M.; Quinn, S. J. Photochem. Photobiol. Sci. 2009, 8, 542.

(24) Matsika, S. J. Phys. Chem. A 2004, 108, 7584.

(25) Matsika, S. J. Phys. Chem. A 2005, 109, 7538.

(26) Improta, R.; Barone, V. Chem. Rev. 2004, 104, 1231.

(27) Zgierski, M. Z.; Patchkovskii, S.; Fujiwara, T.; Lim, E. C. J. Phys. Chem. A 2005, 109,9384

(28) Merchán, M.; Gonzalez-Luque, R.; Climent, T.; Serrano-Andres, L.; Rodriguez, E.; Reguero, M.; Pelaez, D. J. Phys. Chem. B 2006, 110, 26471. 
(29) Hudock, H. R.; Levine, B. G.; Thompson, A. L.; Satzger, H.; Townsend, D.; Gador, N.; Ullrich, S.; Stolow, A.; Martinez, T. J. J. Phys. Chem. A 2007, 111, 8500.

(30) Ismail, N.; Blancafort, L.; Olivucci, M.; Kohler, B.; Robb, M. A. J. Am. Chem. Soc. 2002, 124, 6818.

(31) Blancafort, L.; Robb, M. A. J. Phys. Chem. A 2004, 108, 10609.

(32) Zgierski, M. Z.; Patchkovskii, S.; Lim, E. C. J. Chem. Phys. 2005, 123, 081101.

(33) Zgierski, M. Z.; Alavi, S. Chem. Phys. Lett. 2006, 426, 398.

(34) Blancafort, L. Photochem. Photobiol. 2007, 83, 603.

(35) Kistler, K. A.; Matsika, S. J. Phys. Chem. A 2007, 111, 2650.

(36) Zgierski, M. Z.; Fujiwara, T.; Kofron, W. G.; Lim, E. C. Phys. Chem. Chem. Phys. 2007, 9,3206

(37) Marian, C. M. J. Chem. Phys. 2005, 122, 104314.

(38) Marian, C.; Nolting, D.; Weinkauf, R. Phys. Chem. Chem. Phys. 2005, 7, 3306

(39) Nielsen, S. B.; Theis I. Sølling. ChemPhysChem 2005, 6, 1276.

(40) Perun, S.; Sobolewski, A. L.; Domcke, W. Chem. Phys. 2005, 313, 107.

(41) Perun, S.; Sobolewski, A. L.; Domcke, W. J. Am. Chem. Soc. 2005, 127, 6257

(42) Blancafort, L. J. Am. Chem. Soc. 2006, 128, 210.

(43) Serrano-Andres, L.; Merchán, M.; Borin, A. C. Proc. Natl. Acad. Sci. USA 2006, $103,8691$.

(44) Serrano-Andres, L.; Merchán, M.; Borin, A. C. Chemistry - A European Journal 2006, 12,6559 .

(45) Barbatti, M.; Lischka, H. J. Phys. Chem. A 2007, 111, 2852.

(46) Chung, W. C.; Lan, Z.; Ohtsuki, Y.; Shimakura, N.; Domcke, W.; Fujimura, Y. Phys. Chem. Chem. Phys. 2007, 9, 2075.

(47) Santoro, F.; Barone, V.; Improta, R. Proc. Natl. Acad. Sci. USA 2007, 104, 9931.

(48) Yamazaki, S.; Kato, S. J. Am. Chem. Soc. 2007, 129, 2901.

(49) Langer, H.; Doltsinis, N. L. Phys. Chem. Chem. Phys. 2003, 5, 4516.

(50) Langer, H.; Doltsinis, N. L. Phys. Chem. Chem. Phys. 2004, 6, 2742.

(51) Langer, H.; Doltsinis, N. L.; Marx, D. ChemPhysChem 2005, 6, 1734.

(52) Chen, H.; Li, S. J. Chem. Phys. 2006, 124, 154315.

(53) Zgierski, M. Z.; Patchkovskii, S.; Fujiwara, T.; Lim, E. C. Chem. Phys. Lett. 2007, $440,145$.

(54) Marian, C. M. J. Phys. Chem. A 2007, 111, 1545.

(55) Serrano-Andres, L.; Merchán, M.; Borin, A. C. J. Am. Chem. Soc. 2008, 130, 2473.

(56) Yamazaki, S.; Domcke, W. J. Phys. Chem. A 2008, 112, 7090.

11965.

(57) Yamazaki, S.; Domcke, W.; Sobolewski, A. L. J. Phys. Chem. A 2008, 112,

(58) Shukla, M. K.; Leszczynski, J. J. Phys. Chem. B 2008, 112, 5139.

(59) Lan, Z. G.; Fabiano, E.; Thiel, W. ChemPhysChem 2009, 10, 1225.

(60) Shukla, M. K.; Leszczynski, J. Chem. Phys. Lett. 2009, ASAP.

(61) Canuel, C.; Mons, M.; Piuzzi, F.; Tardivel, B.; Dimicoli, I.; Elhanine, M. J. Chem. Phys. 2005, 122, 0743161.

(62) Karunakaran, V.; Kleinermanns, K.; Improta, R.; Kovalenko, S. A. J. Am. Chem. Soc. 2009, 131, 5839.

(63) Miannay, F.-A.; Banyasz, A.; Gustavsson, T.; Markovitsi, D. J. Am. Chem. Soc. 2007, 129, 14574. 
(64) Miannay, F. A.; Banyasz, A.; Gustavsson, T.; Markovitsi, D. J. Phys. Chem. C 2009, 113, 11760 .

(65) Miles, H. T.; Frazier, J.; Howard, F. B. Science 1963, 142, 1458.

(66) Markovitsi, D.; Onidas, D.; Gustavsson, T.; Talbot, F.; Lazzarotto, E. J. Am.

Chem. Soc. 2005, 127, 17130

(67) Gustavsson, T.; Cassara, L.; Gulbinas, V.; Gurzadyan, G.; Mialocq, J.-C.;

Pommeret, S.; Sorgius, M.; van der Meulen, P. J. Phys. Chem. A 1998, 102, 4229.

(68) Voet, D.; Gratzer, W. B.; Cox, R. A.; Doty, P. Biopolymers 1963, 1, 193.

(69) Clark, L. B. J. Am. Chem. Soc. 1994, 116, 5265.

(70) Bouvier, B.; Gustavsson, T.; Markovitsi, D.; Millié, P. Chem. Phys. 2002, 275, 75.

(71) Carsughi, F.; Ceretti, M.; Mariani, P. European Biophysical Journal 1992, 21, 155.

(72) Lakowicz, J. Principles of Fluorescence Spectroscopy, 3d ed.; Springer-Verlag: New York, 3rd ed., 2006.

(73) Pal, S. K.; Peon, J.; Zewail, A. H. Chem. Phys. Lett. 2002, 363, 57.

(74) Sharonov, A.; Gustavsson, T.; Marguet, S.; Markovitsi, D. Photochem. Photobiol. Sci. 2003, 2, 1 .

(75) Bányász, Á.; Gustavsson, T.; Keszei, E.; Improta, R.; Markovitsi, D. Photochem. Photobiol. Sci. 2008, 7, 765.

(76) Mons, M.; Piuzzi, F.; Dimicoli, I.; Gorb, L.; Leszczynski, J. J. Phys. Chem. A 2006, 110, 10921.

(77) Åberg, U.; Sundström, V. Chem. Phys. Lett. 1991, 185, 461. 


\section{Tables}

Table 1. Fluorescence quantum yields of dGMP in water as a function of excitation wavelength.

\begin{tabular}{|c|c|}
\hline Excitation wavelength $(\mathrm{nm})$ & $\begin{array}{c}\text { Fluorescence quantum yield } \\
\left(\mathrm{x} 10^{4}\right)\end{array}$ \\
\hline 250 & $1.03 \pm 0.07$ \\
\hline 255 & $1.09 \pm 0.10^{\mathrm{a}}$ \\
\hline 265 & $1.31 \pm 0.07$ \\
\hline 275 & $1.45 \pm 0.14$ \\
\hline 285 & $1.51 \pm 0.14$ \\
\hline
\end{tabular}

${ }^{a}$ taken as reference value from ref. 16

Table 2. Time constants (in ps) obtained from separate fits of dGMP fluorescence decays. These correspond to merged data for the individual emission wavelengths. The pre-exponential factors are normalized according to $\sum_{i}\left|\alpha_{i}\right| \equiv 1$

\begin{tabular}{|c|c|c|c|c|c|c|c|c|c|}
\hline$\lambda(\mathrm{nm})$ & $\begin{array}{c}\text { Appfunc } \\
\text { Fwhm }\end{array}$ & $\tau_{\text {RISE }}$ & $\alpha_{\text {RISE }}$ & $\tau_{1}$ & $\alpha_{1}$ & $\tau_{2}$ & $\alpha_{2}$ & $\tau_{3}$ & $\alpha_{3}$ \\
\hline 310 & $0.32 \pm 0.01$ & - & - & 0.16 & 0.87 & 0.94 & 0.13 & & \\
& & & & \pm 0.02 & \pm 0.02 & \pm 0.09 & \pm 0.02 & & \\
\hline 330 & $0.31 \pm 0.01$ & - & - & 0.15 & 0.78 & 0.97 & 0.22 & & \\
& & & & \pm 0.02 & \pm 0.02 & \pm 0.09 & \pm 0.02 & & \\
\hline 360 & $0.28 \pm 0.01$ & - & - & 0.26 & 0.68 & 1.01 & 0.32 & & \\
& & & & \pm 0.02 & \pm 0.04 & \pm 0.08 & \pm 0.04 & & \\
\hline 420 & $0.27 \pm 0.01$ & - & - & 0.29 & 0.36 & 0.69 & 0.51 & $(2.0)$ & 0.13 \\
& & & & \pm 0.08 & \pm 0.17 & \pm 0.14 & \pm 0.14 & & \pm 0.05 \\
\hline 450 & $0.27 \pm 0.01$ & - & - & - & - & 0.62 & 0.80 & 2.2 & 0.20 \\
& & & & & & \pm 0.05 & \pm 0.08 & \pm 0.6 & \pm 0.08 \\
\hline 500 & $0.30 \pm 0.01$ & 0.12 & -0.29 & - & - & 0.73 & 0.42 & 2.13 & 0.29 \\
& & \pm 0.03 & \pm 0.08 & & & \pm 0.09 & \pm 0.05 & \pm 0.16 & \pm 0.05 \\
\hline 600 & $0.30 \pm 0.01$ & 0.16 & -0.49 & - & - & 1.35 & 0.43 & 4.0 & 0.08 \\
& & \pm 0.01 & \pm 0.03 & & & \pm 0.11 & \pm 0.04 & \pm 1.0 & \pm 0.04 \\
\hline
\end{tabular}




\section{Figure Captions}

Figure 1. The steady-state absorption spectrum and fluorescence quantum yields of dGMP for different excitation wavelengths between 250 and $285 \mathrm{~nm}$.

Figure 2. Steady-state fluorescence spectra of dGMP, $\lambda_{\mathrm{exc}}=255$ (blue), 266 (red), and $275 \mathrm{~nm}$ (black). The spectra are scaled by the relative fluorescence quantum yield.

Figure 3. dGMP fluorescence difference spectra constructed from those obtained with excitation at 255 and $275 \mathrm{~nm}$, normalized at the peak (334 nm, blue) or the red edge (700 nm, red).

Figure 4. Experimental fluorescence decays and corresponding fitted curves at 310, 330, 360, 420, 450, 500 and $600 \mathrm{~nm}$. The time-constants of $0.16,0.67$ and 2.0 ps were obtained by a global tri-exponential fit.

Figure 5. Decay Associated Spectra of the three exponential terms in the global tri-exponential fit, corresponding to time constants $0.16 \mathrm{ps}$ (circles), $0.67 \mathrm{ps}$ (triangles) and $2.0 \mathrm{ps}$ (squares).

Figure 6. Fluorescence anisotropy decays of dGMP in water at 330 (blue), 420 (green) and 500 (pink) $\mathrm{nm}$.

Figure 7. Time-resolved fluorescence spectra of dGMP in water.

Figure 8. Time-resolved spectra of dGMP in water at chosen delay times together with the corresponding lognormal fits shown on a wavenumber scale. 
Figure 1

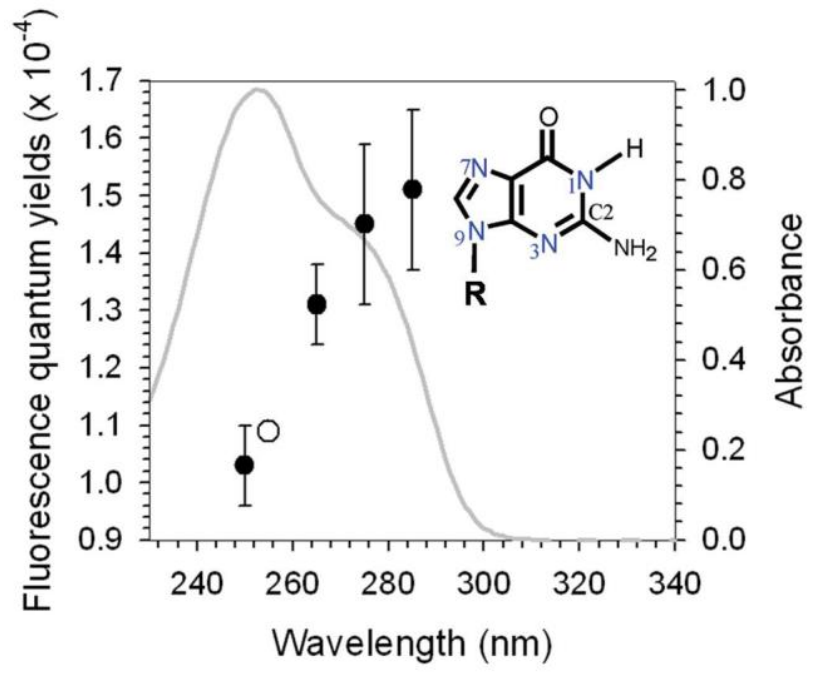

The steady-state absorption spectrum and fluorescence quantum yields of dGMP for different excitation wavelengths between 250 and $285 \mathrm{~nm}$. 
Figure 2

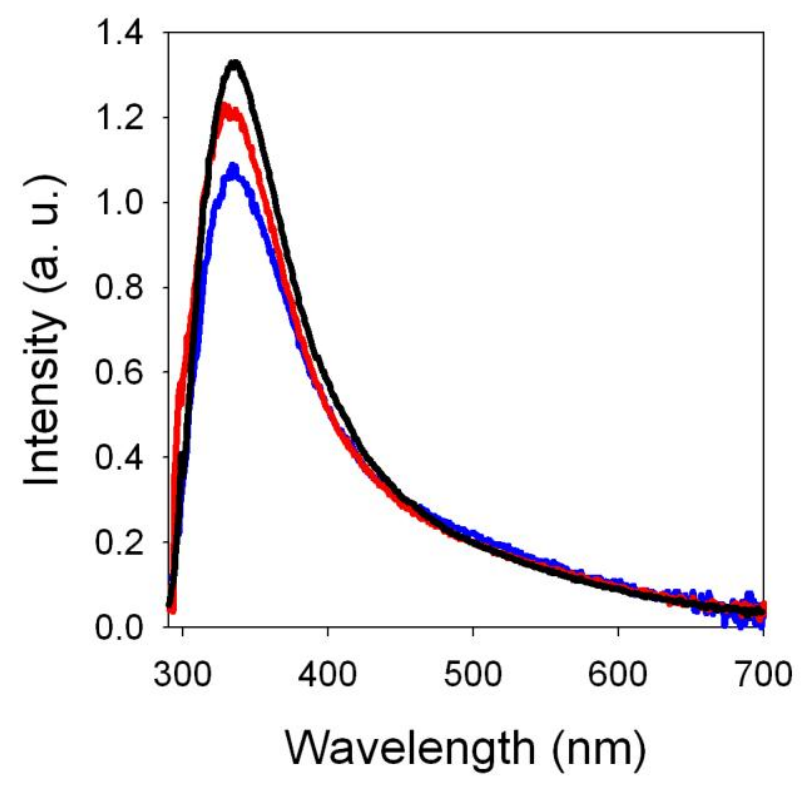

Steady-state fluorescence spectra of dGMP, $\lambda_{\text {exc }}=255$ (blue), 266 (red), and $275 \mathrm{~nm}$ (black). The spectra are scaled by the relative fluorescence quantum yield. 
Figure 3

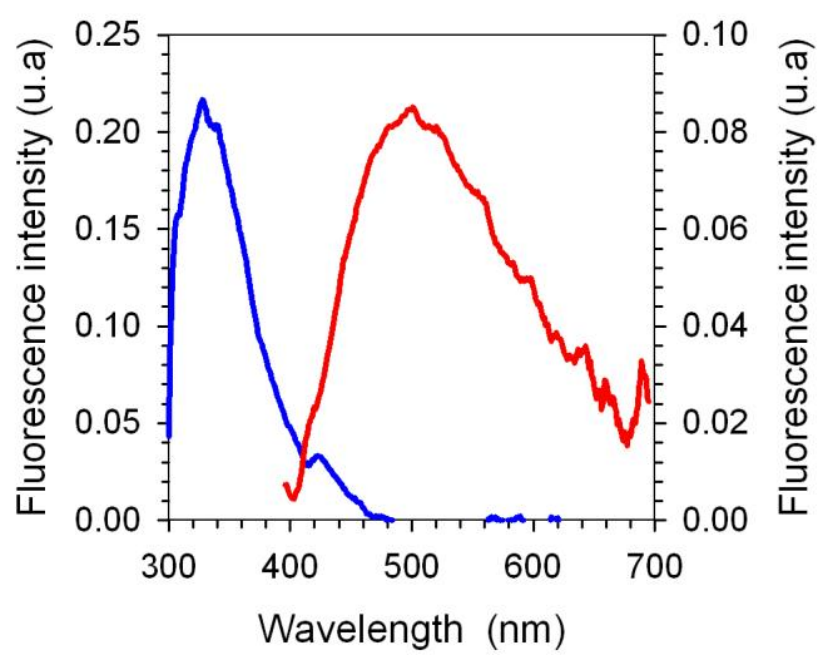

dGMP fluorescence difference spectra constructed from those obtained with excitation at 255 and $275 \mathrm{~nm}$, normalized at the peak (334 nm, blue) or the red edge (700 nm, red). 
Figure 4

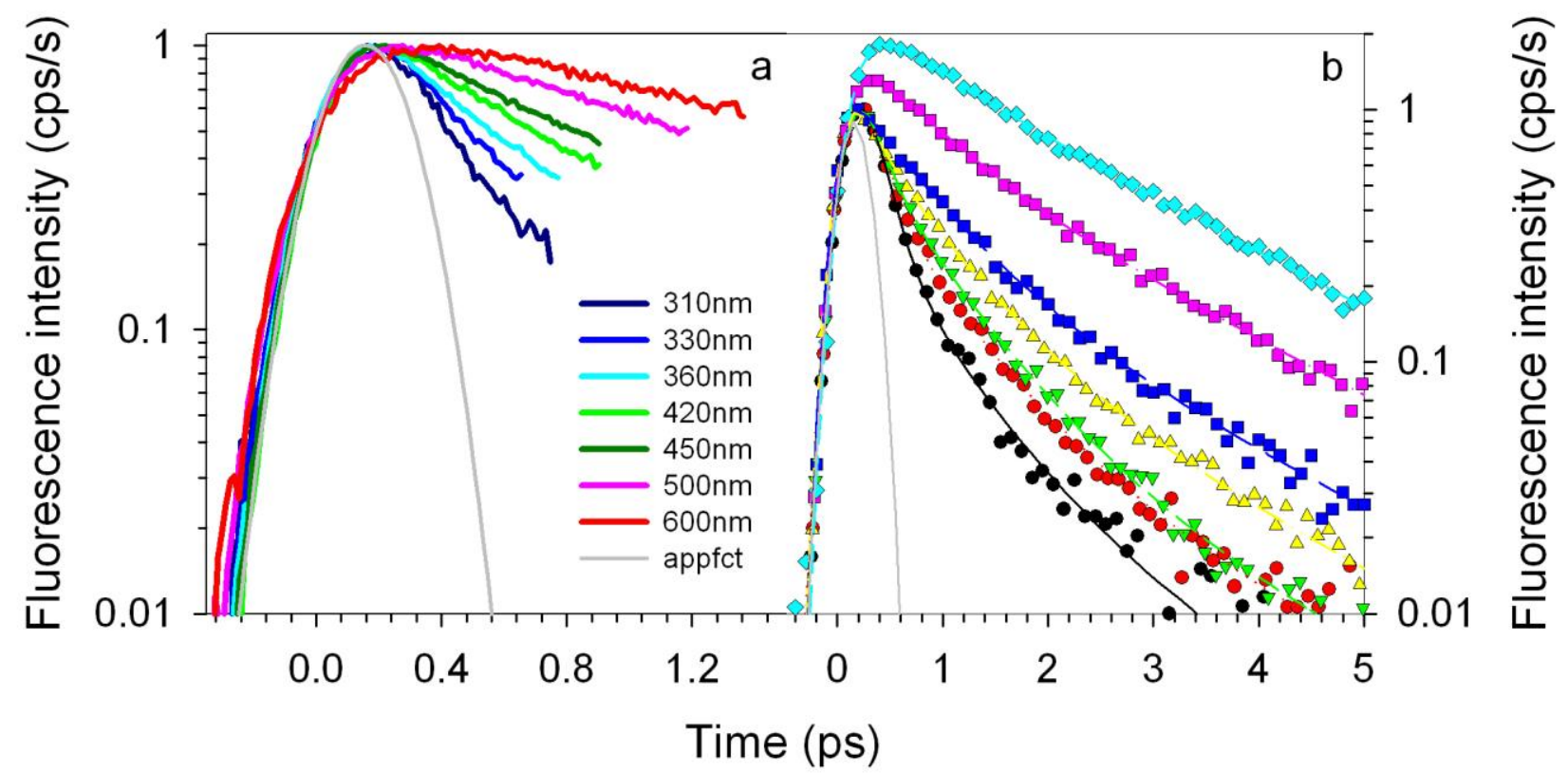

Experimental fluorescence decays and corresponding fitted curves at 310, 330, 360, 420, 450, 500 and $600 \mathrm{~nm}$. The time-constants of $0.16,0.67$ and $2.0 \mathrm{ps}$ were obtained by a global triexponential fit. 
Figure 5

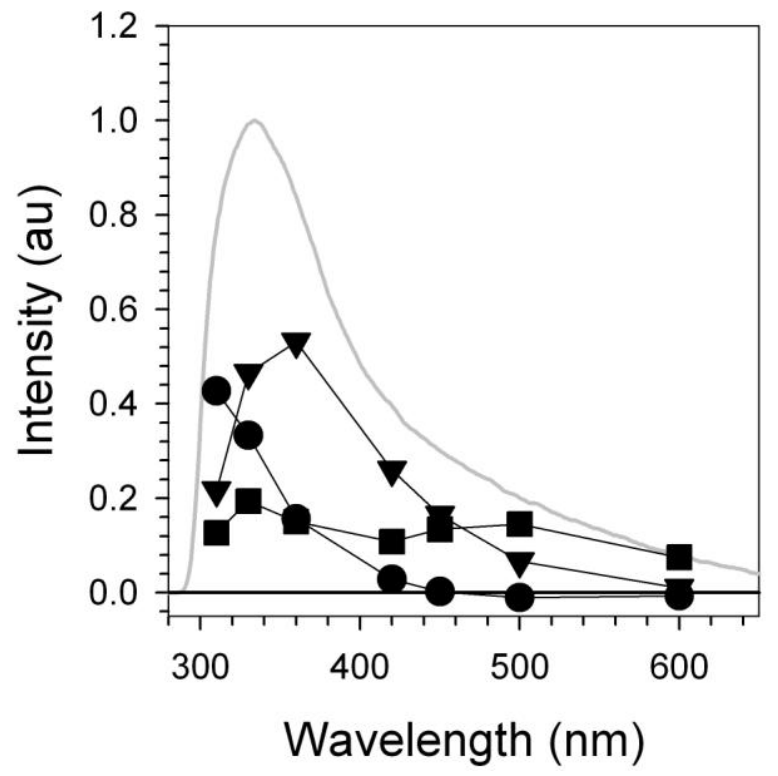

Decay Associated Spectra of the three exponential terms in the global tri-exponential fit, corresponding to time constants $0.16 \mathrm{ps}$ (circles), $0.67 \mathrm{ps}$ (triangles) and $2.0 \mathrm{ps}$ (squares). 
Figure 6

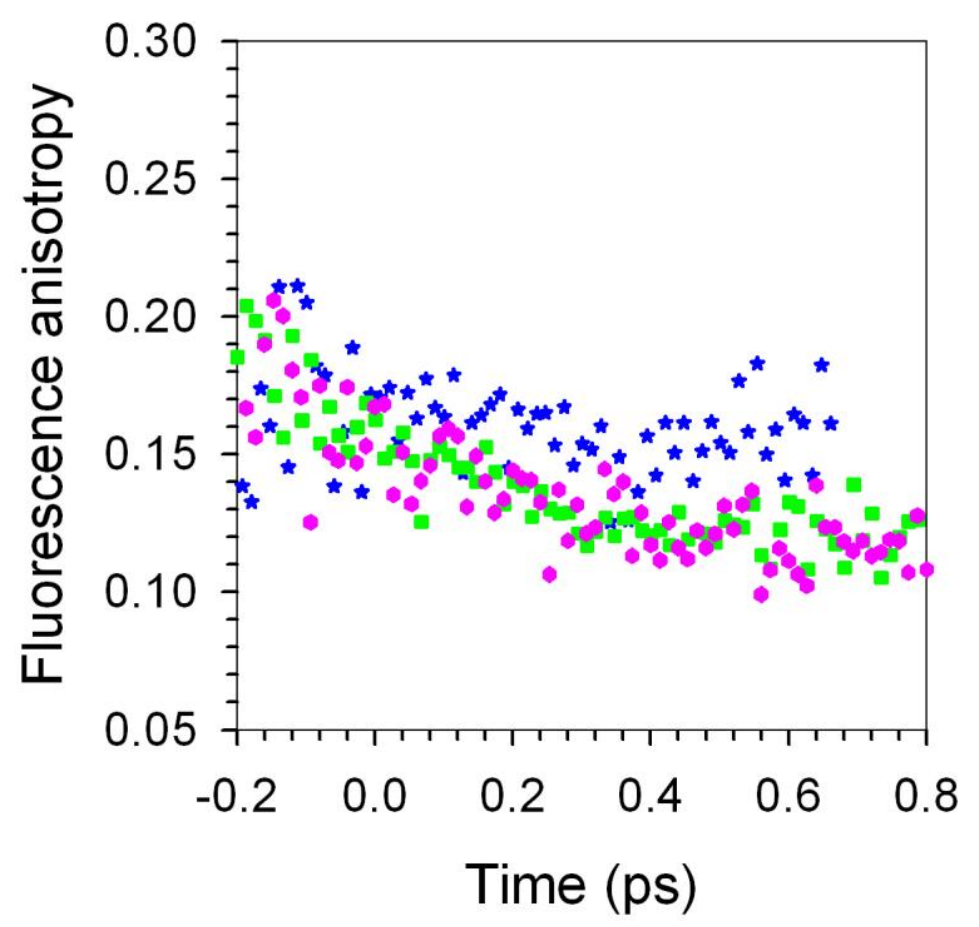

Fluorescence anisotropy decays of dGMP in water at 330 (blue), 420 (green) and 500 (pink) nm. 
Figure 7

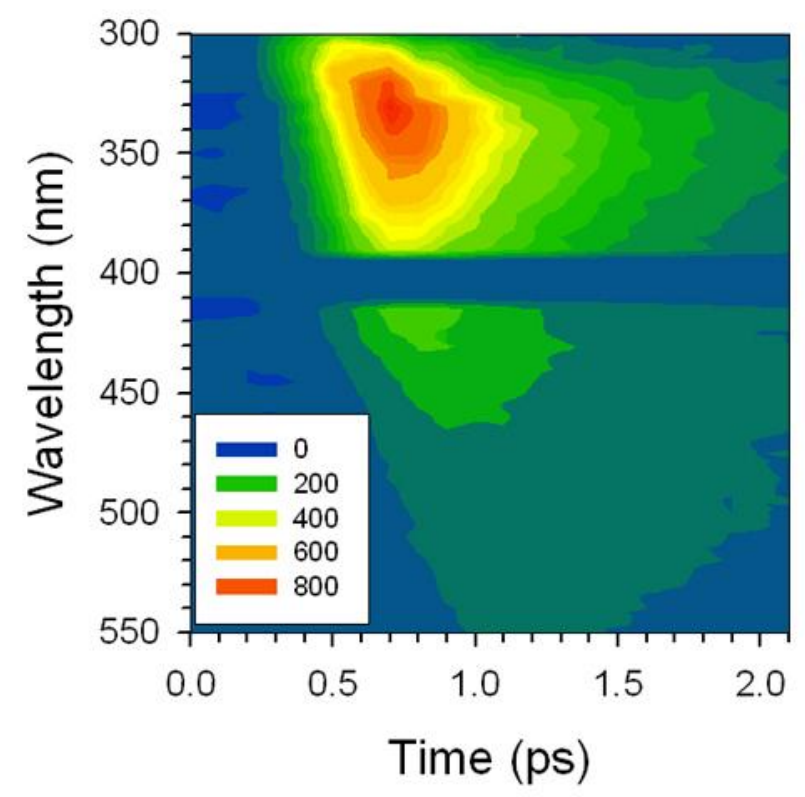

Time-resolved fluorescence spectra of dGMP in water. 
Figure 8

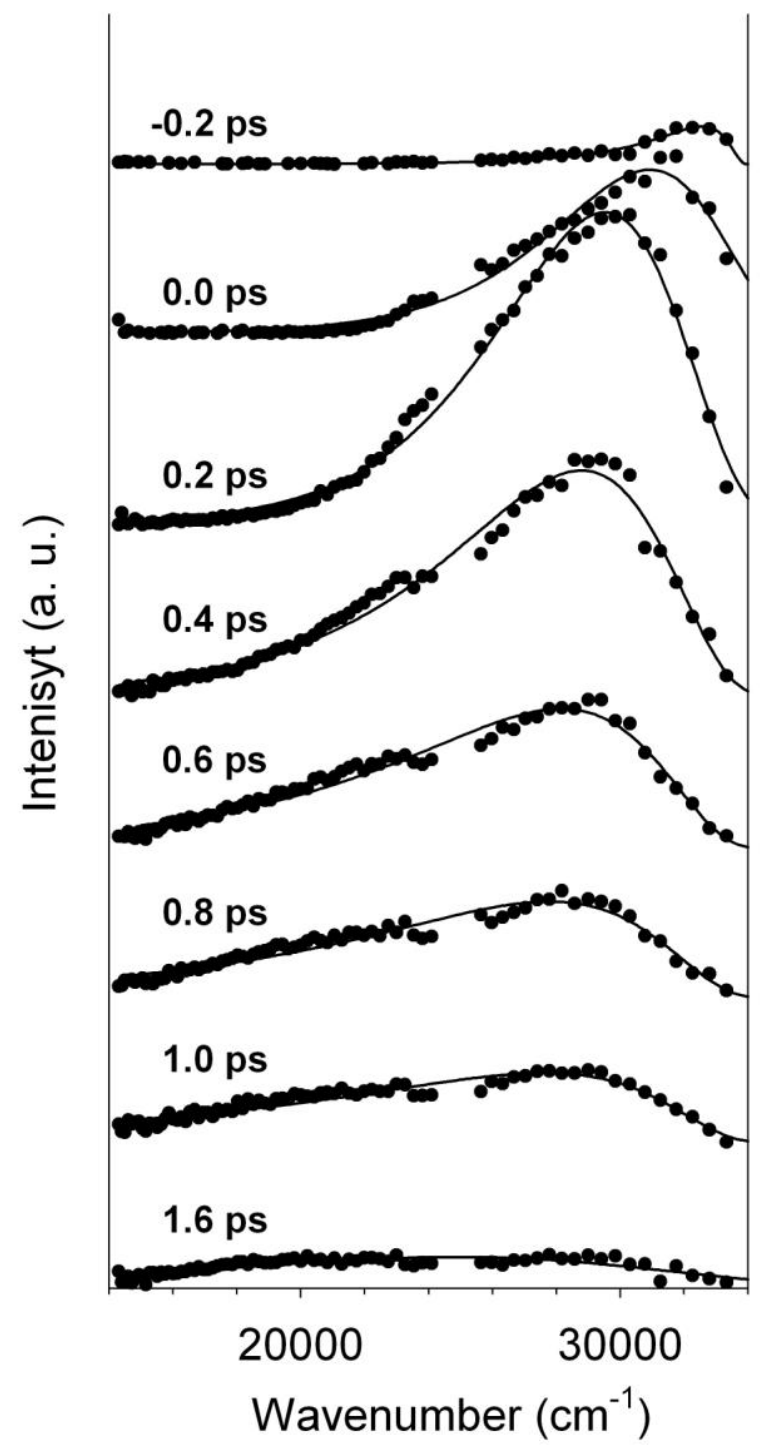

Time-resolved spectra of dGMP in water at chosen delay times together with the corresponding lognormal fits shown on a wavenumber scale. 


\section{TOC Figure}

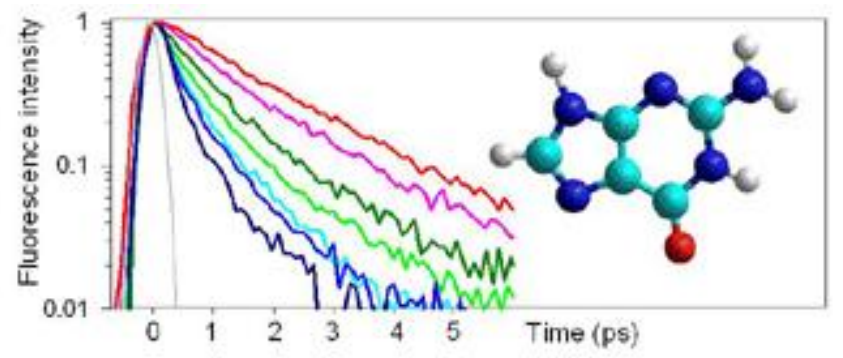

